\title{
Sinking Skin Flap Syndrome after Hemicraniectomy and Ventriculo-Peritoneal Shunt Overdrainage
}

Christiana Ossig ${ }^{1^{*}}$, Claudia Lindner ${ }^{2}$, Johannes Gerber ${ }^{3}$ and Jochen Schaefer ${ }^{1}$

${ }^{1}$ Neurology, Medizinische Fakultat Carl Gustav Carus, Technische Universitaet Dresden, Fetscherstraße 74, 01307 Dresden, Germany

${ }^{2}$ Neurosurgery, Medizinische Fakultät Carl Gustav Carus, Technische Universitaet Dresden, Fetscherstraße 74, 01307 Dresden, Germany

${ }^{3}$ Neuroradiology, Medizinische Fakultät Carl Gustav Carus, Technische Universitaet Dresden, Fetscherstraße 74, 01307 Dresden, Germany

*Corresponding author: Dr. med. Christiana Ossig, Department of Neuropsychiatry and Laboratory of Molecular Psychiatry, Charité- Universitaetsmedizin Berlin, 10117 Berlin, Germany, Tel: 0049-351-458-2532; Fax: 0049-351-458-5802; E-mail: christiana.ossig@uniklinikum-dresden.de

Received date: Dec 10, 2014, Accepted date: Feb 17, 2015, Published date: Mar 24, 2015

Copyright: ( 2015 Ossig C, et al. This is an open-access article distributed under the terms of the Creative Commons Attribution License, which permits unrestricted use, distribution, and reproduction in any medium, provided the original author and source are credited.

\begin{abstract}
We present a case of sinking skin flap syndrome after hemicraniectomy and insertion of a ventriculo-peritoneal shunt following severe craniocerebral injury. When the neurological status deteriorated due to cerebrospinal fluid overdrainage and resulting midline shift, complete ligation of the vp shunt became a temporary solution before definitive cranioplasty could be performed.
\end{abstract}

Keywords: Sinking skin flap syndrome; vp shunt; Shunt overdrainage; Craniectomy; Cranioplasty

\section{Introduction}

The sinking skin flap syndrome is a rare complication after hemicraniectomy and is characterized by the occurrence of headache, epileptic seizures, vertigo, dysesthesia, palsy and reduced vigilance $[1,2]$. It is considered to be caused at the site of the defect by atmospheric pressure, reduced cerebral blood flow (CBF) and altered cerebrospinal fluid (CSF)-pressure - or a combination thereof [1]. Atmospheric pressure may lead to direct cortical compression [2]. Improvement of $\mathrm{CBF}$ at the site of the craniectomy and the opposite hemisphere following cranioplasty has been shown by radiological studies including xenon computertomography $(\mathrm{CT})$, perfusion $\mathrm{CT}$, and dynamic CT [3-5]. A decrease in CSF pressure, due to changes in posture, but also caused by overdrainage after shunt insertion, may result in collapse of brain structure $[6,7,1]$.

\section{Case report}

A 46-year-old man suffered a brain injury with left-sided subdural and epidural hematoma, requiring left-sided decompressive hemicraniectomy. In addition, a ventriculo-peritoneal (vp) shunt was inserted because of posttraumatic obstructive hydrocephalus using a valve-opening pressure (vop) of $130 \mathrm{mmH}_{2} \mathrm{O}$. Clinically, the patient showed a residual hemiparesis and right-sided hemispasticity. Three months after the accident cranioplasty was performed. Two years later, the cranioplasty dislocated with a skull depression of $2 \mathrm{~cm}$ and had to be removed. After refixation, an intracranial large hygroma developed and consequently the vop was increased to $200 \mathrm{mmH}_{2} \mathrm{O}$. Subsequently, an epidural abscess developed and the cranioplasty had to be removed. One week later, the patient presented with a fluctuating level of consciousness and was admitted to our hospital.

On examination, the patient was somnolent and showed a marked right-sided hemiparesis and tetraspasticity. On admission, a CT scan revealed a slit left lateral ventricle and compression of the right lateral ventricle due to midline shift (Figure 1). We suspected overdrainage of the vp shunt and after interdisciplinary discussion complete surgical ligation of the vp shunt was performed leading to major clinical improvement: the patient was awake and was able to interact with his environment using single words and head movements. A follow-up CT scan showed ventricular expansion on the left and reduced midline shift (Figure 2). The patient could be discharged and completed his rehabilitation treatment, before he underwent cranioplasty 6 weeks later (Figure 3). The timepoint for cranioplasty was chosen in regard to the previous epidural abscess and the clinical condition of the patient. During this procedure the ligation was removed and the vop was again set at $200 \mathrm{~mm} \mathrm{H}_{2} \mathrm{O}$.

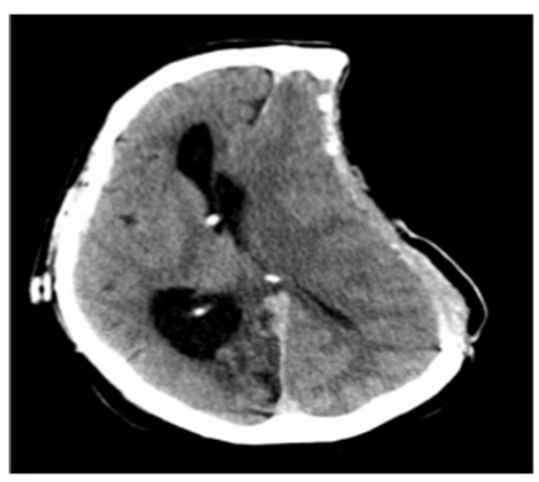

Figure 1: CT Scan on admission 
Citation: Ossig C, Lindner C, Gerber J, Schaefer J (2015) Sinking Skin Flap Syndrome after Hemicraniectomy and Ventriculo-Peritoneal Shunt Overdrainage. J Neurol Neurophysiol 6: 272. doi:10.4172/2155-9562.1000272

Page 2 of 2

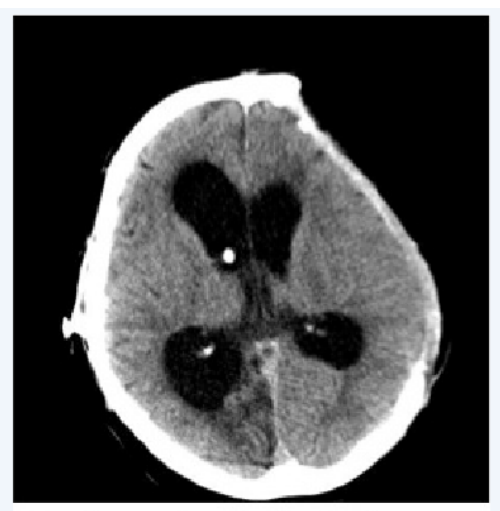

Figure 2: CT Scan 2 days after shunt ligation

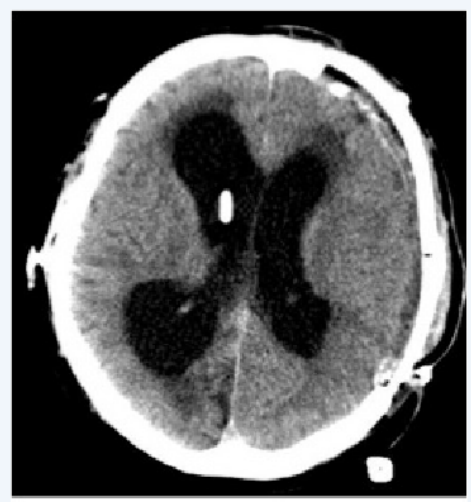

Figure 3: CT Scan after cranioplasty

\section{Discussion}

Vp shunt overdrainage and thus compression of the brain by atmospheric pressure following large hemicraniectomy led to neurological deterioration in our patient. This phenomenon is known as sinking skin flap syndrome (SSFS). The pathogenesis of SSFS in our patient is supported by the observation that only complete surgical ligation of the vp shunt reverted the midline shift and normalized the patient's level of consciousness. The evidence-based treatment of this syndrome is prompt cranioplasty [8]. In our case, this procedure could not be performed because of the previous infection. Therefore, ligation of the shunt was performed as a temporary solution and resulted in clinical improvement. Slow reduction of the vop is further planned in order to decrease the ventricle size.

\section{References}

1. Stula D (1982). The problem of the "sinking skin-flap syndrome" in cranioplasty. J Maxillofac Surg 10: 142-145.

2. Yamaura A, Makino H (1977). Neurological deficits in the presence of the sinking skin flap following decompressive craniectomy. Neurol Med Chir 17: 43-53.

3. Sakamoto S, Eguchi K, Kiura Y, Arita K, Kurisu K (2006). CT perfusion imaging in the syndrome of the sinking skin flap before and after cranioplasty [Case Reports]. Clin Neurol Neurosurg 108: 583-585.

4. Suzuki N, Suzuki S, \& Iwabuchi T (1993). Neurological improvement after cranioplasty. Analysis by dynamic CT scan. Acta Neurochir 122: 49-53.

5. Wintermark M, Thiran JP, Maeder P, Schnyder P, Meuli R (2001). Simultaneous measurement of regional cerebral blood flow by perfusion CT and stable xenon CT: a validation study. AJNR Am J Neuroradiol, 22: 905-914.

6. Dujovny M, Agner C, Aviles A (1999). Syndrome of the trephined: theory and facts. Crit Rev Neurosurg, 9: 271-278.

7. Fodstad H, Love JA, Ekstedt J, Friden H, Liliequist B (1984). Effect of cranioplasty on cerebrospinal fluid hydrodynamics in patients with the syndrome of the trephined. [Research Support, Non-U.S. Gov't]. Acta Neurochir (Wien) 70: 21-30.

8. Yamaura A, Sato M, Meguro K, Nakamura T, Uemura K (1977). [Cranioplasty following decompressive craniectomy--analysis of 300 cases (author's transl)]. [Case Reports]. No Shinkei Geka 5: 345-353. 